\title{
IRREGULARITY OF MOLECULAR GRAPHS
}

\section{Ivan Gutman}

Faculty of Science, University of Kragujevac, P. O. Box 60, 34000 Kragujevac, Serbia, and State University of Novi Pazar, Novi Pazar, Serbia e-mail: gutman@kg.ac.rs

(Received February 10, 2016)

ABSTRACT. A graph whose all vertices have equal degrees is said to be regular. If this is not the case, then the graph is irregular. Various measure of irregularity have been proposed. These are described and compared, with particular emphasis on molecular graphs.

\section{Introduction}

Let $G=(V, E)$ be a (molecular) graph with vertex set $V=V(G)$ and edge set $E=E(G)$, having $n=|V(G)|$ vertices and $m=|E(G)|$ edges $[26,32]$. Let $V(G)=$ $\left\{v_{1}, v_{2}, \ldots, v_{n}\right\}$. For $v_{i} \in V(G)$, the degree of the vertex $v_{i}$, denoted by $d\left(v_{i}\right)=d_{G}\left(v_{i}\right)$, is the number of vertices adjacent to $v$. The degree sequence of the graph $G$ is the non-increasing sequence of its degrees,

$$
\mathbf{d}=\mathbf{d}(G)=\left[d\left(v_{1}\right), d\left(v_{2}\right), \ldots, d\left(v_{n}\right)\right]
$$

such that

$$
d\left(v_{1}\right) \geq d\left(v_{2}\right) \geq \cdots \geq d\left(v_{n}\right) .
$$

At this point we recall that

$$
\sum_{v \in V(G)} d_{G}(v)=2 m
$$


The number of vertices of the graph $G$ whose degree is equal to $i$ will be denoted by $n_{i}=n_{i}(G)$. Then, of course,

$$
\sum_{i=1}^{n-1} n_{i}=n
$$

and according to Eq. (2),

$$
\sum_{i=1}^{n-1} i n_{i}=2 m .
$$

A graph $G$ is is said to be regular if all its vertices have the same degree. Regular graphs played an outstanding role in the history of graph theory [11] and are still in the focus of interest of mathematicians. This is seen from the fact that numerous regular graphs have special names, such as the Petersen graph, Moore graph, Hoffman-Singleton graph, Shrikhande graph, Klein graph, Hall-Janko graph, Schläfli graph, etc. Important and much studied is the class of "strongly regular graphs". In mathematical chemistry, regular graphs occur has much less frequently. Only after the the discovery of fullerenes and nanotubes, such graphs started to attract the attention of mathematical chemists. Irrespective of this, the fact is that the vast majority of molecular graphs are non-regular.

A graph which is not regular is said to be irregular.

It seems that Pál Erdös ${ }^{1}$ was the first who recognized that it is reasonable to ask about how irregular a non-regular graph is. In 1988, Erdős et al. published a paper [12] in which they asked "Which class of graphs is opposite to the regular graphs?" Such "opposite" graphs should be highly irregular. During the Second Krakow Conference on Graph Theory (1994), Erdős officially posed as an open problem the determination of the extreme size of highly irregular graphs of given order [40]. Eventually, highly irregular graphs were examined in $[6,39,40]$. The degree sequence of highly irregular graphs was studied in [39].

Evidently, a graph would be maximally irregular if all its degrees would differ, i.e., if

$$
d\left(v_{1}\right)>d\left(v_{2}\right)>\cdots>d\left(v_{n}\right)
$$

Graphs with this property were named "perfect". However, Behzad and Chartrand [9]

\footnotetext{
${ }^{1}$ Pál Erdős (1913-1996), Hungarian mathematician, one of the most outstanding and most prolific mathematicians of the 20th century.
} 
established that perfect graphs do not exist. What does exist are the "quasi-perfect" graphs (sometimes referred to as "antiregular graphs" [43]). These are graphs in which all vertices, except two, have different degrees. The respective result is [9]:

Theorem 1.1. [9] For each $n>1$, there is a unique connected quasi-perfect graph of order $n$. Its degree sequence is

$$
\mathbf{d}=[n-1, n-2, \ldots,\lfloor n / 2\rfloor,\lfloor n / 2\rfloor, \ldots, 2,1]
$$

Note that the complement of a quasi-perfect graph is also quasi-perfect. However, the complement of a connected quasi-perfect graph is disconnected, and is therefore of no relevance for the present considerations.

Of these quasi-perfect graphs only those depicted in Fig. 1 are molecular graphs.

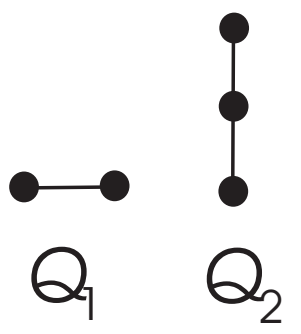

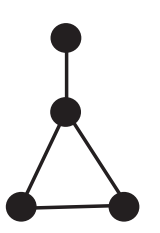

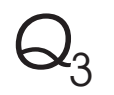

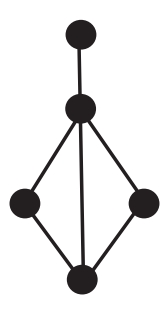

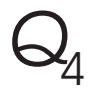

Fig. 1. The first four connected quasi-perfect graphs. With the exception of $Q_{1}$, these may be considered as the most irregular molecular graphs.

\section{$2 \quad$ Irregularity index}

A simple and straightforward way of expressing the irregularity of a graph is via its irregularity index, $\operatorname{Irr}_{\mathbf{d}}$, equal to the the number of distinct elements in the degree sequence, or in a more formal notation:

$$
\operatorname{Irr}_{\mathbf{d}}=\left|\left\{i \mid n_{i}>0\right\}\right|
$$

This concept was, in an implicit manner, used in the early works $[5,6,39,40]$, but was explicitly considered only quite recently $[36,37,41,42]$. 
In the case of molecular graphs, the irregularity index should be applied with due caution, or - better - not applied at all. Namely, by counting only the number of different values in the degree sequence $\mathbf{d}(G)$, Eq. (1), we disregard the number of times each value occurs. Even worse, we also disregard the existence (or nonexistence) of pairs of adjacent vertices of different degrees. Two self-explanatory examples of these difficulties are shown in Fig. 2.
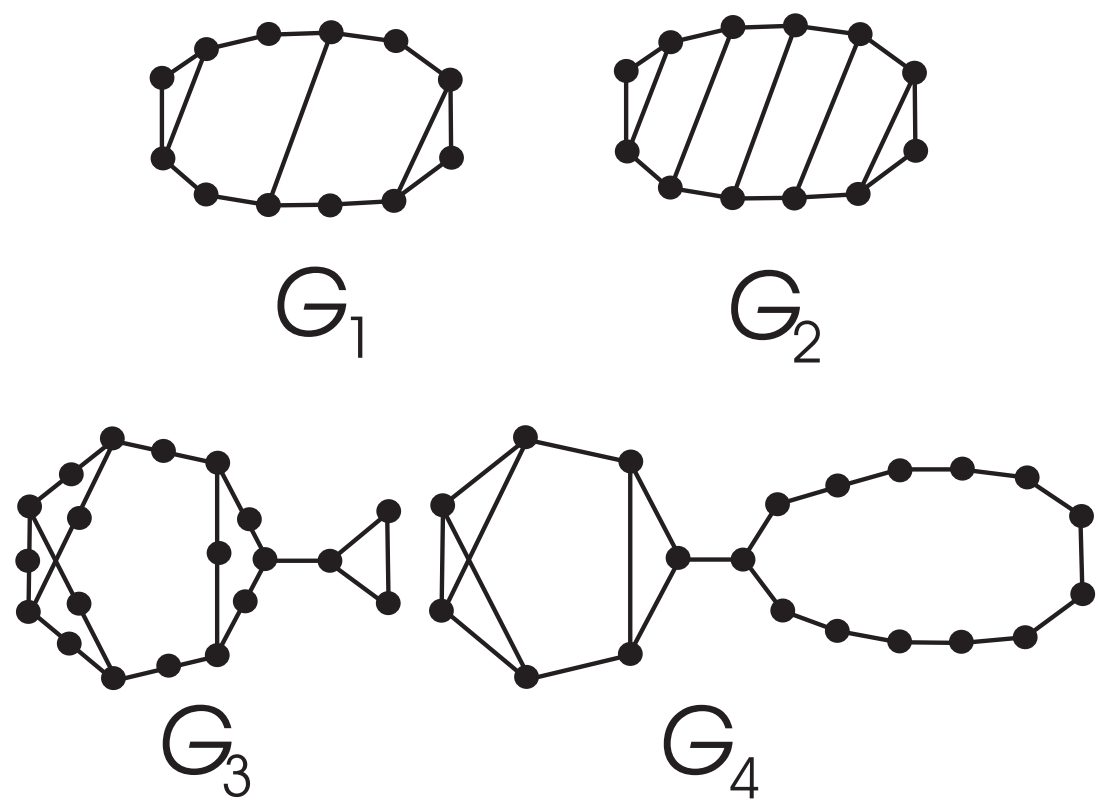

Fig. 2. Two pairs of graphs illustrating the weakness of the concept of irregularity index $\operatorname{Irr}_{\mathbf{d}}$. For details see text.

In Fig. 2 are depicted two pairs of graphs, having equal irregularity indices $\operatorname{Irr}_{\mathbf{d}}$.

The graphs $G_{1}$ and $G_{2}$ each have 12 vertices, some of degree two, some of degree three. Therefore, the $\operatorname{Irr}_{\mathbf{d}}$-values of $G_{1}$ and $G_{2}$ are equal, $\operatorname{Irr}_{\mathbf{d}}\left(G_{1}\right)=\operatorname{Irr}_{\mathbf{d}}\left(G_{2}\right)=2$. Yet, in $G_{1}$ the number of degree two and degree three vertices is equal, $n_{2}\left(G_{1}\right)=$ $n_{3}\left(G_{1}\right)=6$. Contrary to this, the graph $G_{2}$ is almost regular, since all but two of its vertices are of degree three, $n_{2}\left(G_{2}\right)=2, n_{3}\left(G_{2}\right)=10$. Intuitively, one would expect that $G_{1}$ is much more irregular than $G_{2}$.

The graphs $G_{3}$ and $G_{4}$ each have 20 vertices, some of degree two, some of degree three. Therefore, also the $\operatorname{Irr}_{\mathbf{d}}$-values of $G_{3}$ and $G_{4}$ are equal, $\operatorname{Irr}_{\mathbf{d}}\left(G_{3}\right)=\operatorname{Irr}_{\mathbf{d}}\left(G_{4}\right)=$ 
2. The number of degree two vertices in $G_{3}$ and $G_{4}$ are equal, $n_{2}\left(G_{3}\right)=n_{2}\left(G_{4}\right)=12$ and the same is the case with degree three vertices, $n_{3}\left(G_{3}\right)=n_{3}\left(G_{4}\right)=8$, i.e., $\mathbf{d}\left(G_{3}\right)=\mathbf{d}\left(G_{4}\right)$. Yet, in $G_{3}$ all but two edges connect a degree two and a degree three vertex. Contrary to this, $G_{4}$ may be viewed as consisting of a regular graph of degree three joined by an edge to a regular graph of degree two. Intuitively, one would expect that $G_{3}$ is much more irregular than $G_{4}$.

\section{Structure--dependent irregularity indices}

Any measure of graph irregularity, say $\operatorname{Irr}(G)$, must satisfy the following requirements:

$(\alpha) \operatorname{Irr}(G)=0$ if and only if the (connected) graph $G$ is regular.

$(\beta) \operatorname{Irr}(G)>0$ if the (connected) graph $G$ is not regular.

The irregularity index $\operatorname{Irr}_{\mathbf{d}}$ described above, satisfies these requirements. However, bearing in mind the difficulties explained in the preceding section, another requirement for $\operatorname{Irr}$ needs to be added:

$(\gamma)$ The quantity $\operatorname{Irr}$ has to be defined so that its numerical value follows our intuitive feeling for "deviating from regularity".

It seems that the first such measure of graph irregularity is found in the seminal work of Collatz and Sinogowits [14], who proved that $\lambda_{1}$, the greatest eigenvalue of the adjacency matrix, satisfies the inequality $\lambda_{1} \geq 2 \mathrm{~m} / n$. In the case of connected graphs, equality occurs if and only if this graph is regular. Thus, the Collatz-Sinogowitz irregularity measure is

$$
\operatorname{Irr}_{C S}=\lambda_{i}-\frac{2 m}{n}
$$

Its application is not easy, because the $\lambda_{1}$-value of a (non-regular) graph $G$ cannot be directly deduced from the structure of $G$ (see, for instance, $[45,48]$ ).

Certain irregularity measures depend solely on the degree sequence $\mathbf{d}$, and can thus be written as $\operatorname{Irr}=f(\mathbf{d})$. These automatically imply that two graphs with equal degree sequence are equally irregular. In particular, according to such irregularity 
measures, the graphs $G_{3}$ and $G_{4}$ in Fig. 2 would be claimed to be equally irregular.

The simplest and best known index of this kind is that of Bell [10]. He measured the irregularity of a graph by means of the variance of its vertex degrees. Bell's irregularity index is

$$
\operatorname{Irr}_{\text {Bell }}(G)=\frac{1}{n} \sum_{v \in V(G)}\left(d(v)-\frac{2 m}{n}\right)^{2}=\frac{1}{n} \sum_{i=1}^{n-1} n_{i}\left(i-\frac{2 m}{n}\right)^{2}
$$

recalling that the average value of vertex degrees is $2 m / n$.

Also the below mentioned $\operatorname{Irr}_{t o t 2}$, Eq. (4), belongs to this class, cf. Eq. (5).

Another direction of approaching the irregularity is by taking into account the difference of degrees of adjacent vertices. By this, two graphs with equal degree sequence need not necessarily be considered as equally irregular. In particular, the irregularity of the graphs $G_{3}$ and $G_{4}$ from Fig. 2 will be found to differ.

Thus, for an edge $e=u v \in E(G)$, the difference considered is $\left|d_{G}(u)-d_{G}(v)\right|[8,13]$. Accordingly, Albertson conceived the irregularity of a graph $G$ as [7]

$$
\operatorname{Irr}_{A l b}=\sum_{u v \in E(G)}\left|d_{G}(u)-d_{G}(v)\right|
$$

which is usually referred to as the Albertson index [22,24,38,44,49,50], although the name "third Zagreb index" has also been proposed [21]. A similar, yet not much studied quantity would be [23]

$$
\operatorname{Irr}_{A l b 2}(G)=\sum_{u v \in E(G)}\left[d_{G}(u)-d_{G}(v)\right]^{2}
$$

Recently [1,3], the total irregularity was introduced, defined as

$$
I r r_{t o t}=\sum_{\{u, v\} \subseteq V(G)}\left|d_{G}(u)-d_{G}(v)\right|
$$

which in analogy with (3) could be modified as

$$
\operatorname{Irr}_{t o t 2}=\sum_{\{u, v\} \subseteq V(G)}\left[d_{G}(u)-d_{G}(v)\right]^{2}
$$

Some of the above specified irregularity measures are related to the two Zagreb indices $M_{1}(G)$ and $M_{2}(G)$, and the $F$-index $[23,25,27,28,30,47]$ 


$$
\begin{aligned}
M_{1}(G) & =\sum_{v \in V(G)} d_{G}(v)^{2}=\sum_{i=1}^{n-1} i^{2} n_{i} \\
M_{2}(G) & =\sum_{u v \in E(G)} d_{G}(u) d_{G}(v) \\
F(G) & =\sum_{v \in V(G)} d_{G}(v)^{3}=\sum_{i=1}^{n-1} i^{3} n_{i} .
\end{aligned}
$$

Namely,

$$
\begin{aligned}
\operatorname{Irr}_{\text {Bell }} & =\frac{1}{n} M_{1}(G)-\left(\frac{2 m}{n}\right)^{2} \\
\operatorname{Irr}_{A l b 2} & =F(G)-2 M_{2}(G) \\
\operatorname{Irr}_{\text {tot } 2} & =n M_{1}(G)-4 m^{2}
\end{aligned}
$$

For comparative studies of $\operatorname{Irr}_{C S}, \operatorname{Irr}_{A l b}, \operatorname{Irr}_{t o t}$, and $\operatorname{Ir} r_{\text {Bell }}$ see $[10,15,17,31]$. For more information on the above specified irregularity measures see the papers $[1-4,16,17,23,33-35,46]$ and the references cited therein. For additional irregularity measures see [18-20,29].

\section{Concluding remarks}

The concept of "graph irregularity", i.e., the extent by which a graph deviates from "regularity" is a notion that has a vague meaning. From our everyday's experience we have some "intuitive" feeling about this notion, and in some cases the majority of us would agree that a particular graph is more "irregular" than another. (For examples, see Fig. 2.) When one attempts to quantify this concept, and give a precise method for its measuring, then we encounter ambiguity. Simply: measures of "graph irregularity" can be constructed in numerous agreeable, but different, ways. In this survey we presented a dozen of such measures, whereas a skilled scholar could easily design a dozen more.

Needless to say that this arbitrariness is an opportunity to produce a multitude of papers that resemble serious scientific research. 
"Graph irregularity" is not the only such scientific conundrum. Analogous situations are encountered with "complexity", "cyclicity", "branching", to mention just a few.

\section{REFERENCES}

[1] H. Abdo, S. Brandt, D. Dimitrov, The total irregularity of a graph, Discr. Math. Theor. Comput. Sci. 16 (2014) 201-206.

[2] H. Abdo, N. Cohen, D. Dimitrov, Graphs with maximal irregularity, Filomat 28 (2014) 1315-1322.

[3] H. Abdo, D. Dimitrov, The total irregularity of graphs under graph operations, Miskolc Math. Notes 15 (2014) 3-17.

[4] H. Abdo, D. Dimitrov, The irregularity of graphs under graph operations, Discuss. Math. Graph Theory 34 (2014) 263-278.

[5] Y. Alavi, A. Boals, G. Chartrand, P. Erdős, O. Oellermann, k-path irregular graphs, Congr. Numer. 65 (1988) 201-210.

[6] Y. Alavi, G. Chartrand, F. R. K. Chung, P. Erdős, R. L. Graham, O. R. Oellermann, Highly irregular graphs, J. Graph Theory 11 (1987) 235-249.

[7] M. O. Albertson, The irregularity of a graph, Ars Comb. 46 (1997) 219-225.

[8] M. Albertson, D. Berman, Ramsey graphs without repeated degrees, Congr. Numer. 83 (1991) 91-96.

[9] M. Behzad, G. Chartrand, No graph is perfect, Am. Math. Monthly 74 (1967) 962-963.

[10] F. K. Bell, A note on the irregularity of graphs, Lin. Algebra Appl. 161 (1992) $45-54$.

[11] N. L. Biggs, E. K. Lloyd, R. J. Wilson, Graph Theory 1736 - 1936, Clarendon Press, Oxford, 1976.

[12] G. Chartrand, P. Erdős, O. Oellermann, How to define an irregular graph, College Math. J. 19 (1988) 36-42. 
[13] G. Chen, P. Erdős, C. Rousseau, R. Schelp, Ramsey problems involving degrees in edge-colored complete graphs of vertices belonging to monochromatic subgraphs, Eur. J. Comb. 14 (1993) 183-189.

[14] L. Collatz, U. Sinogowitz, Spektren endlicher Graphen, Abh. Math. Sem. Univ. Hamburg 21 (1957) 63-77.

[15] D. Cvetković, P. Rowlinson, On connected graphs with maximal index, Publ. Inst. Math. Beograd 44 (1988) 29-34.

[16] D. Dimitrov, T. Réti, Graphs with equal irregularity indices, Acta Polytechn. Hung. 11(4) (2014) 41-57.

[17] D. Dimitrov, R. Škrekovski, Comparing the irregularity and the total irregularity of graphs, Ars Math. Contemp. 9 (2015) 45-50.

[18] C. S. Edwards, The largest vertex degree sum for a triangle in a graph, Bull. London Math. Soc. 9 (1977) 203-208.

[19] C. Elphick, P. Wocjan, New measures of graph irregularity, El. J. Graph Theory Appl. 2(1) (2014) 52-65.

[20] E. Estrada, Randić index, irregularity and complex biomolecular networks, Acta Chim. Slov. 57 (2010) 597-603.

[21] G. Fath-Tabar, Old and new Zagreb indices of graphs, MATCH Commun. Math. Comput. Chem. 65 (2011) 79-84.

[22] G. H. Fath-Tabar, I. Gutman, R. Nasiri, Extremely irregular trees, Bull. Acad. Serbe Sci. Arts (Cl. Sci. Math. Natur.) 145 (2013) 1-8.

[23] B. Furtula, I. Gutman, A forgotten topological index, J. Math. Chem. 53 (2015) 1184-1190.

[24] B. Furtula, I. Gutman, M. Dehmer, On structure-sensitivity of degree-based topological indices, Appl. Math. Comput. 219 (2013) 8973-8978.

[25] B. Furtula, I. Gutman, Ž. Kovijanić Vukićević, G. Lekishvili, G. Popivoda, On an old/new degree-based topological index, Bull. Acad. Serbe Sci. Arts (Cl. Sci. Math. Natur.) 148 (2015) 19-31.

[26] I. Gutman, Introduction to Chemical Graph Theory, Univ. Kragujevac, Kragujevac, 2003 (in Serbian). 
[27] I. Gutman, Degree-based topological indices, Croat. Chem. Acta 86 (2013) 351361.

[28] I. Gutman, On the origin of two degree-based topological indices, Bull. Acad. Serbe Sci. Arts (Cl. Sci. Math. Natur.) 146 (2014) 39-52.

[29] I. Gutman, B. Furtula, C. Elphick, Three new/old vertex-degree-based topological indices, MATCH Commun. Math. Comput. Chem. 72 (2014) 617-632.

[30] I. Gutman, B. Furtula, Ž. Kovijanić Vukićević, G. Popivoda, On Zagreb indices and coindices, MATCH Commun. Math. Comput. Chem. 74 (2015) 5-16.

[31] I. Gutman, P. Hansen, H. Mélot, Variable neighborhood search for extremal graphs 10. Comparison of irregularity indices for chemical trees, J. Chem. Inf. Model. 45 (2005) 222-230.

[32] I. Gutman, O. E. Polansky, Mathrmatical Concepts in Organic Chemistry, Springer, Berlin, 1986.

[33] A. Hamzeh, T. Réti, An analogue of Zagreb index inequality obtained from graph irregularity measures, MATCH Commun. Math. Comput. Chem. 72 (2014) 669684.

[34] P. Hansen, H. Mélot, Variable neighborhood search for extremal graphs. 9. Bounding the irregularity of a graph, in: S. Fajtlowicz, P. W. Fowler, P. Hansen, M. F. Janowitz, F. S. Roberts (Eds.), Graphs and Discovery, American Math. Soc., Providence, 2005, pp. 253-264.

[35] M. Henning, D. Rautenbach, On the irregularity of bipartite graphs, Discr. Math. 307 (2007) 1467-1472.

[36] B. Horoldagva, L. Buyantogtokh, S. Dorjsembe, I. Gutman, Maximum size of maximally irregular graphs, MATCH Commun. Math. Comput. Chem. 76 (2016), in press.

[37] F. Liu, Z. Zhang, J. Meng, The size of maximally irregular graphs and maximally irregular triangle-free graphs, Graphs Comb. 30 (2014) 699-705.

[38] W. Luo, B. Zhou, On the irregularity of trees and unicyclic graphs with given matching number, Util. Math. 83 (2010) 141-148.

[39] Z. Majcher, J. Michael, Degree sequence of highly irregular graphs, Discr. Math. 164 (1997) 225-236. 
[40] Z. Majcher, J. Michael, Highly irregular graphs with extreme numbers of edges, Discr. Math. 164 (1997) 237-242.

[41] S. Mukwembi, A note on diameter and the degree sequence of a graph, Appl. Math. Lett. 25 (2012) 175-178.

[42] S. Mukwembi, On the maximally irregular graphs, Bull. Malays. Math. Sci. Soc. 36 (2013) 717-721.

[43] E. Munarini, Characteristic, admittance and matching polynomial of an antiregular graph, Appl. Anal. Discr. Math. 3 (2009) 157-176.

[44] R. Nasiri, G. H. Fath-Tabar, The second minimum of the irregularity of graphs, El. Notes Discr. Math. 45 (2014) 133-140.

[45] O. E. Polansky, I. Gutman, On the calculation of the largest eigenvalue of molecular graph, MATCH Commun. Math. Comput. Chem. 5 (1979) 149-159.

[46] T. Réti, D. Dimitrov, On irregularity of bidegreed graphs, Acta Polytechn. Hung. 10(7) (2013) 117-134.

[47] D. Stevanović, Mathematical Properties of Zagreb Indices, Akademska misao, Beograd, 2014 (in Serbian).

[48] D. Stevanović, Spectral Radius of Graphs, Elsevier, Amsterdam, 2015.

[49] M. Tavakoli, F. Rahbarnia, M. Mirzavaziri, A. R. Ashrafi, I. Gutman, Extremely irregular graphs, Kragujevac J. Math. 37 (2013) 135-139.

[50] B. Zhou. W. Luo, On irregularity of graphs, Ars Comb. 88 (2008) 55-64. 